\title{
Managing Sensor Data in Ambient Assisted Living
}

\author{
C. D. Nugent* , L. Galway, L. Chen, and M. P. Donnelly \\ Faculty of Computing and Engineering, School of Computing and Mathematics, University of Ulster, Jordanstown, UK \\ cd.nugent@ulster.ac.uk, I.galway@ulster.ac.uk, l.chen@ulster.ac.uk, mp.donnelly@ulster.ac.uk
}

\section{S. I. McClean, S. Zhang, B. W. Scotney, and G. Parr}

Faculty of Computing and Engineering, School of Computing and Information Engineering, University of Ulster, Coleraine, UK

si.mcclean@ulster.ac.uk, s.zhang@ulster.ac.uk, bw.scotney@ulster.ac.uk, gp.parr@ulster.ac.uk

\begin{abstract}
The use of technology within the home has gained wide spread acceptance as one possible approach to be used in addressing the challenges of an ageing society. A number of rudimentary assistive solutions are now being deployed in real settings but with the introduction of these technology-orientated services come a number of challenges, which to date are still largely unsolved. At a fundamental level, the management and processing of the large quantities of data generated from multiple sensors is recognised as one of the most significant challenges. This paper aims to present an overview of the types of sensor technologies used within Ambient Assisted Living. Subsequently, through presentation of a series of case studies, the paper will demonstrate how the practical integration of multiple sources of sensor data can be used to improve the overall concept and applications of Ambient Assisted Living.
\end{abstract}

Category: Smart and intelligent computing

Keywords: Sensor data; Assistive technologies; Ambinet assisted living; Smart homes; Smart environments

\section{INTRODUCTION}

The advances that have been made in today's society from economic, social and medical perspectives can be considered as factors contributing to global ageing. Current estimates predict that both the size and age profile of the population will change significantly within the next half century. United Nations estimates suggest that the world's population will increase to close to 8.9 billion within the next 40 years. This is close to a $50 \%$ increase since the year 2000. In terms of the age profile within the population, in virtually every country we have witnessed the group of those aged 60 and over to be the fastest growing. Correspondingly, estimates from the World Health Organisation (WHO) suggest that by 2050, the number of older people on a global scale will have increased to 2 billion. This is approximately a 3-fold increase on the figure of 600 million older people in the year 2000 [1].
These changes in population have been accompanied by an increase in the prevalence of those suffering from long term chronic diseases, in addition to a larger number of older people that wish to live within their own homes for a longer period of time. As a result, increased burdens are now being placed upon health and social care provision, delivery of informal care and national pension schemes. Consequently, attempts to surmount the challenges posed by the necessity for a revised healthcare model have led to the introduction of new paradigms in the delivery of both health and social care. Amongst these, the use of Information and Communication Technology (ICT) has gained much interest and, indeed, success. The widespread adoption of affordable technologies, coupled with improvements in processing and communication speeds, are all key factors in technology-based solutions being investigated further.

This paper aims to provide an insight into the use of sensorbased technology to support Ambient Assisted Living (AAL). 
Specifically, its focus will be on the complexities of managing different sources of sensor-based information with the overall goal of improving the levels of automated support within an AAL context. The remainder of the paper is structured as follows: Section II presents an overview of the range of sensorbased technologies used within the smart home paradigm. Section III introduces the inherent requirements for appropriate processing infrastructures that are capable of managing data from differing data sources. Two case studies are presented within Section III in order to further elaborate upon these concepts. Section IV concludes the paper with a discussion on potential future directions within this domain and scope for further work.

\section{OVERVIEW OF SENSOR BASED TEHCNOLOGIES}

Technology has been a core component of healthcare provision for more than a century. Since the introduction of computing technology to healthcare in 1957 by Pipberger as a means of processing the electrocardiogram (ECG) [2], computer-based technology has now embedded itself firmly within hospital, community and home healthcare settings. To a large extent this can be attributed to decreases in the cost of technology coupled with increases in processing power and the availability of data storage, along with the prevalent acceptance and adoption of the Internet and both fixed and wireless modes of communications.

The AAL Program aims to define key aspects which should be taken into consideration when deciding how ICT should be used in order to support home-based healthcare [3, 4]. Through a series of consolidated efforts, a Program of Work has been established which recognises there is an opportunity to improve the quality of life for older people through the use of ICT. This involves the creation of smarter environments at home, at work and in the community through the combination of products, services and systems. Fig. 1 provides a representation of what may be interpreted as the main factors which contribute to the AAL Program.

\section{A. Technology within the Smart Home}

In its simplest form, we can represent a smart home as having 3 main components; sensors, a central data processing component and an environmental control component (Fig. 2). The role

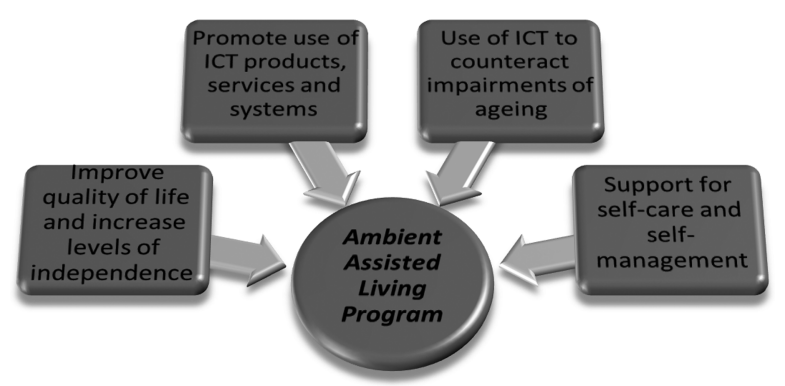

Fig. 1. Overview of the main factors contributing to the Ambient Assisted Living Program [3, 4]. ICT: Information and Communication Technology.

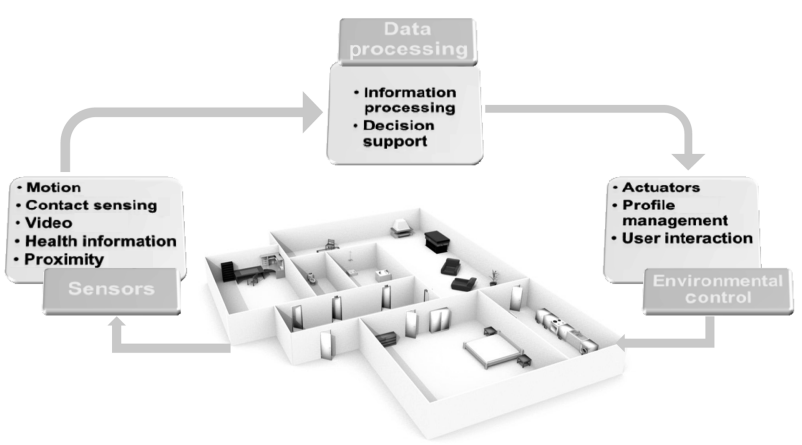

Fig. 2. The Smart Home Paradigm with the three main components of sensors, data processing and environment control [5].

of the sensor technology is to acquire information from the environment in order to provide, for example, details about the location of the inhabitant(s) and the object(s) they interact with. The sensor technology may also be utilised for the acquisition of data from any health-related devices that the inhabitant may have. The data processing component is required to process data gleaned from the sensors in an effort to understand the exact behavior of the inhabitant along with their wellbeing status. Based on this understanding, the environmental control component is used to control the environment in order to provide a form of feedback to the inhabitant and, if required, to assist them somehow with the activity they are trying to undertake [5, 6].

Taking into account the potential positive impact such an environment can have, a wide range of both demonstration and operational environments have been created and evaluated with the goal to support the ageing in such a place [7, 8]. Although progress towards the larger scale deployment of smart home environments has been made in recent years, a number of challenges still exist within each of the three aforementioned main components. From a sensor technology perspective, there remain issues pertaining to the quality of the sensor data, together with trade-offs associated with obtaining the correct balance between the number of sensors to include in an environment and their associated costs. Likewise, from the perspective of data processing, challenges remain in producing reliable assessments of the inhabitant's behavior. In terms of environmental control, the appropriate form of user interaction in order to provide feedback still remains a key issue to be tackled [9]. Amongst all of these challenges, improving the quality of the sensor data upon which the behavioral analysis is based is viewed by some as being one of the most important unresolved issues within this domain.

\section{B. Sensor Technology}

By utilising sensor technologies, potentially, a wide range of measurements can be acquired. This information may be acquired through sensors that are seamlessly embedded within the environment, or it may be acquired through devices the inhabitant is wearing or interacts with $[6,10]$. The measurement of vital signs is perhaps one of the most common forms of measurement that can be recorded remotely in the home environment. Acquiring vital signs can provide useful information for the assessment of health in addition to being an important 
parameter for use in the analysis of long-term trends. The typical type of information acquired can involve the recording of parameters such as temperature, blood pressure, weight, electrocardiogram, heart rate and pulse oximetry. A number of studies have been conducted which have validated the concept of home-based vital signs recording [10,11], and in particular have demonstrated the utility of taking such information into consideration during the management of specific long-term chronic conditions, for example chronic heart failure and diabetes. The sensing technology required for such measurements can come in the form of discrete devices that the inhabitant interacts with, or alternatively they may be in the form of wearable devices that have the ability to take ambulatory measurements [12].

As well as the recording of vital signs, a number of worn devices have the ability to collect measurements of activity. A low level of activity is considered to be one of the key factors associated with health problems within society. Pedometers can be used to assess levels of activity in the form of counting steps [13]. More recently, technology with embedded accelerometers, for example smart phones, have been utilised in order to gain an insight into an inhabitant's level of activity in addition to attempting to identify which activity the inhabitant is engaged in [14].

Assistive technologies such as cognitive prosthetics, medication management devices and fall detectors can all provide further levels of information relating to an inhabitant's behavior and wellbeing [6]. These can all be considered as discrete sensing devices in their own right with no requirement for further attachment to any additional sensor technologies. Such assistive technologies have the ability to relay information in relation to their usage once they have been interacted with.

With technology that is embedded seamlessly into the environment, one of its most common uses is to assess Activities of Daily Living (ADL). The assessment of a person's ability to undertake ADLs, for example grooming, eating and using the telephone, can be used as a basic measurement of health and wellbeing [15]. One of the most common forms of sensor technology that has been used to record object interactions associated with ADLs is the use of anonymous binary sensors. These sensors can act as simple contact interfaces that can be configured to transmit a short message, for example once an object has been moved or if a door has been opened. Alternative technology in the form of Radio Frequency Identification (RFID) has also been used for similar purposes [15]. Using this approach, objects are tagged with small RFID tags and an RFID reader, which may be worn on the inhabitant's wrist, are utilised to detect any interactions between the object and the inhabitant.

Along with consideration of the objects the inhabitant is interacting with, additional contextual information can be gleaned from identification of the specific location of the inhabitant within their environment. Indoor localisation techniques can be achieved through Infra Red, ultrasound, Bluetooth, Radio Frequency, WiFi and RFID [16, 17]. Nevertheless, indoor localisation solutions vary widely in terms of range, accuracy, cost and the underlying technology platform, hence the correct balance between all of these factors should be taken into account during the design of a smart home environment.

To a certain extent, the use of video-based information can be

\begin{tabular}{|c|c|c|c|c|}
\hline & Video data & Sensor data & Activity data & Physiological data \\
\hline 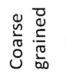 & Person tracking & $\begin{array}{c}\text { Object } \\
\text { interaction }\end{array}$ & Activity levels & Vital signs \\
\hline 总 & Motion analysis & $\begin{array}{l}\text { Time and } \\
\text { sequence } \\
\text { processing }\end{array}$ & $\begin{array}{c}\text { Activity } \\
\text { classification }\end{array}$ & Trend analysis \\
\hline
\end{tabular}

Fig. 3. Management of information collected within the smart home environment.

viewed as one of the only approaches capable of gaining a true insight into which inhabitant is currently within the environment and which activity they are currently engaged in. Nevertheless, providing such an accurate level of service requires a large number of video cameras placed in unobstructed locations throughout the environment. In addition, the processing of visual information typically necessitates elaborate video processing techniques, which may be required to handle large amounts of data in realtime.

\section{Data Analysis}

Based on the level of detail recorded from the sensor technology, analysis performed within the data processing component of the smart home can be provided at two differing levels, as detailed in Fig. 3. In the first instance, coarse grained analysis can provide a general assessment of the inhabitant's behavior, for example determining whether the inhabitant is active or inactive, or having a specific vital sign measurement within a specific range. In the second instance, fine grained analysis of the information collected can provide a more detailed insight into the behavior of the inhabitant. For example, once determined as being active, the analysis can identify if the inhabitant has been simply walking through the house or has been pacing due to high levels of anxiety. Once the assessment has been made, the data processing component should then deliver a form of intervention. This may be in the form of automated activity assistance, for example, an audio prompt to assist with the next sub-task for completing an ADL, or it may involve the transmission of a warning message to a carer or healthcare professional indicating that a further level of assistance is required within the home environment.

It is beyond the scope of this paper to provide a detailed assessment of the specific challenges which exist with each type of sensor based technology presented and their subsequent analysis. Nevertheless, one issue of particular interest is how the information from multiple sensor technologies can be combined in a practical manner to improve the overall assessment of a user's behavior.

\section{MANAGING INFORMATION FROM MULTIPLE SENSORS}

Under ideal circumstances sensor based technology, as presented in the previous Section, can provide sufficient informa- 
tion to gain an insight into the inhabitant's behavior and wellbeing. Nevertheless, in many circumstances this information can be utilised, that is, it is not possible for the data processing component to provide accurate analysis of the data provided. These situations may occur in instances of multiple occupancy when more than one person is in the same room interacting with various objects at the same time, when interleaved activities occur, for example when a person is preparing a meal and uses the telephone at the same time, and when the information acquired from sensors is corrupted by noise or missing data.

There exists a trend at present where the level of information processing has moved beyond that of only considering information from one sensor source in isolation, to a more practical framework that can manage and process multiple sources of sensor information. As a result, more elaborate and reliable service delivery will become possible. These services will have the capability to address practical challenges in both managing and delivering care within smart environments. In our current work we propose a series of practical approaches whereby multiple sources of sensor data can be taken into consideration in an effort to increase the levels of reliability of service delivery. The following subsections present two case studies in an effort to elaborate these concepts further.

\section{A. Case Study 1: Contact Sensors with Video Processing}

In this scenario, we consider a rudimentary smart environment that has a number of contact sensors installed in order to support the recording of information related to which object an inhabitant interacts with whilst undertaking a series of ADLs. Specifically, we wish to consider if it is possible to manage instances of multiple occupancy within the sensor equipped environment by complementing the basic installation of sensor technology with vision processing techniques. Fig. 4 depicts the environment utilised during the experiments, along with exemplars of the sensor technology employed and how they were attached to various objects within the environment [18].

Consider the information presented in Table 1. The information recorded in the first three columns is representative of the data generated automatically by the contact sensors upon activation. Each time a sensor is activated, for example when a cupboard door is opened or a cup is lifted, a message is sent automatically from the sensor to a nearby receiving unit. The

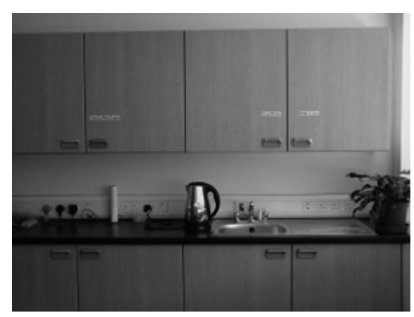

(a)

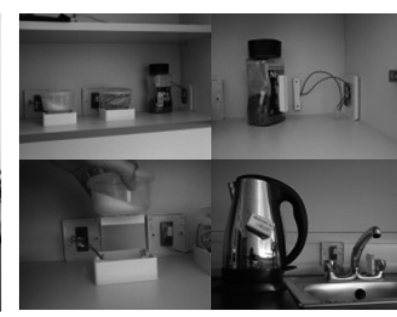

(b)
Fig. 4. Experimental setup (a) Smart kitchen environment used for experiments [18] and (b) examples of contact sensors connected to various objects within the kitchen.
Annotation column in Table 1 provides additional evidence that has been annotated by a human observer during the experiments. The role of the observer is to create a gold standard for the experiment, both in terms of recording which objects were interacted with, and which inhabitants interacted with the objects in each specific instance. This provides the necessary detail relating to the observed instances of multiple occupancy within the environment. For example, Events 1 and 2, as presented in Table 1, have recorded that the cupboard door was opened and a cup was lifted from the cupboard. Under ideal conditions (and if the sensors have been placed appropriately within the environment on the correct objects), the form of sensor technology employed within the experiments will provide adequate information for the data processing component to be able to infer the inhabitant's ADL. Nevertheless, we consider the instance of multiple occupancy where two inhabitants are interacting with various objects within the environment at the same point in time. Consider Events 5 and 6, as presented in Table 1. In this scenario, the fridge door has been opened followed by the cupboard door being opened. From the perspective of the data processing component, based on the information from the contact sensors, there is insufficient evidence to either indicate that this is an instance of multiple occupancy, or even to indicate which inhabitant has interacted with which object. If, on the other hand, there were some way to include information related to each of the multiple occupants individually, this would have a significantly positive impact on the overall processing of the data recorded.

In an effort to improve the accuracy with which object interactions are detected, the contact sensor-based information may be augmented by further sources of sensor-based information. In this Case Study, video was utilised as the secondary source of information. From a practical perspective, the inclusion of a low-cost camera within the kitchen environment is a reasonable additional technical component. The basic concept for processing the video acquired is based around the detection of motion within the recorded scene. One example of how this could be performed would be with a non-adaptive background subtraction technique. This would involve utilising a frame differencing approach in conjunction with the edge detection of objects within the scene. Such a method would have a number of limitations in that it would have to assume nearly constant lighting

Table 1. Excerpt of data from sensors within smart kitchen

\begin{tabular}{ccccccc}
\hline Event & Date & Time & ID & Annotation & Multiple & ID \\
\hline 1 & $29 / 06 / 11$ & 09.53 & 2092 & Person 1 & N & 2092 \\
2 & $29 / 06 / 11$ & 09.54 & 2093 & Person 1 & N & 2093 \\
& & & & & & \\
3 & $29 / 06 / 11$ & 10.45 & 2986 & Person 2 & N & 2986 \\
4 & $29 / 06 / 11$ & 10.47 & 2010 & Person 2 & N & 2010 \\
& & & & & & \\
5 & $29 / 06 / 11$ & 11.32 & 2087 & Person 1 & Y & 2087 \\
6 & $29 / 06 / 11$ & 11.34 & 2093 & Person 2 & Y & 2093 \\
\hline
\end{tabular}

2092: cupboard door open, 2093: cup lifted, 2087: fridge door open, 2077: milk carton lifted, 2010: kettle lifted, 2986: tap on. 


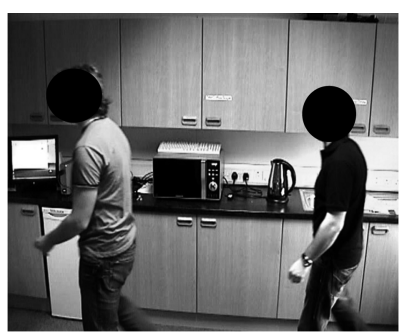

(a)

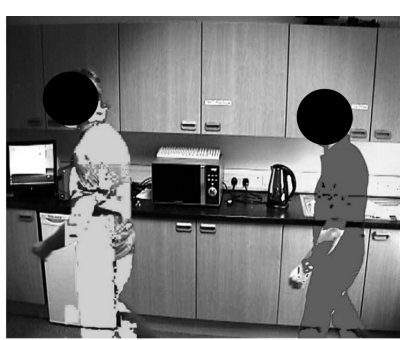

(b)
Fig. 5. Video processing will be capable of taking the original video sequence as shown in (a) and computing areas of motion representative of humans as shown in (b).

conditions, in addition to the need to retaining a static model of the environment. Instances of multiple occupancy could therefore be detected by classifying all areas identified as motion based on their size and profile, as shown in Fig. 5. Subsequently, multiple inhabitants could be both identified and tracked by computing RGB histograms of the classified areas of motion. By taking into account this additional information produced by the video processing component, it is possible to augment the contact sensor information with further details relating to the number of inhabitants within the environment, along with information related to the specific times that they were in differing regions in the environment. Based on this information, a correlation can be made with the object interactions recorded from the contact sensors. For example, if a person was detected as being in the vicinity of the sink (as identified by the video processing component) at the time the tap was turned on (as identified by a contact sensor), these two pieces of sensor information would be deemed to be correlated.

The last two columns in Table 1 provide details of the output from the video processing component. This provides additional information relating to instances of multiple occupancy and information relating to the objects that were used during interactions by each inhabitant. Both sources of information can now be considered by the overall data processing component. In this example we could assume that Person 1 may be the actual inhabitant of the environment and Person 2 may be an informal carer or family visitor.

Full details of an evaluation based on this approach can be found in [19]. The results from that study indicated that each sensor-based approach used in isolation had a number of drawbacks; however, when both were considered together overall improvements in inference were notable in instances of multiple occupancy.

\section{B. Case Study 2: Vital Signs and Activity Levels}

Many previously studied approaches have considered the monitoring of vital signs within the home $[10,11]$. Subsequently, this has led to the development of both new data acquisition devices and wearable devices in the form of smart garments [20]. These approaches have demonstrated their utility in the monitoring and management of health conditions, however, on occassion recordings taken out of context may lead to the provision of spurious data. For example, if heart rate is measured after ascending a set of stairs in a house, its value will be

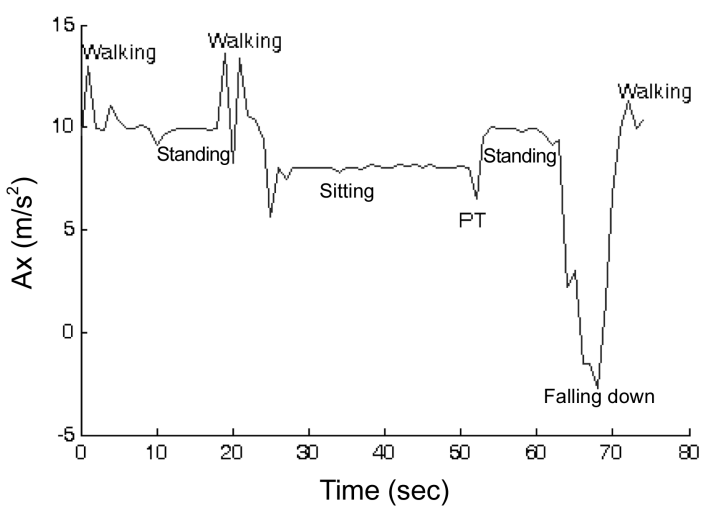

Fig. 6. Acceleration signal from a smart phone annotated for various activities within the home environment. PT: posture transition.

much higher than if the inhabitant had been resting for a 30 minute period prior to taking the measurement.

In this scenario, the framework we present is one that incorporates both vital sign information and activity-related information, with an overarching goal of attempting to improve the level of understanding of the health condition of the inhabitant being monitored. Recently, there has been interest in the use of worn accelerometers within smart environments for the purposes of monitoring levels of activity. Such devices can be utilised to measure both levels of activity, in terms of being active and not active, and can also provide a more detailed assessment of a task an inhabitant is undertaking, for example sitting, standing, walking and lying down. Fig. 6 presents an annotated example of an accelerometer signal recorded from a smart phone as a person undertook the following sequence of activities: walking, standing, walking, sitting, standing, falling down, walking. Sufficient advances have been made in the development of processing modules so that we have the capability to automatically classify a person's behavior with a high degree of accuracy based on acceleration data [14]. Nevertheless, there is still much room for improvement with these approaches.

If we consider the range of heart rate values obtained over a period of time for a given person, we can hypothesize that a change in heart rate is correlated with some other external factor that may be measurable through assessment of the subject's activity. Fig. 7 presents an example of a person's heart rate changing over a period of time. Based solely on this information, the only assessment that can be made is that the heart rate has changed significantly during this period but no further explanations as to the cause of this change can be provided.

In an effort to address this challenge and to gain a deeper understanding of unknown effects related to the change of heart

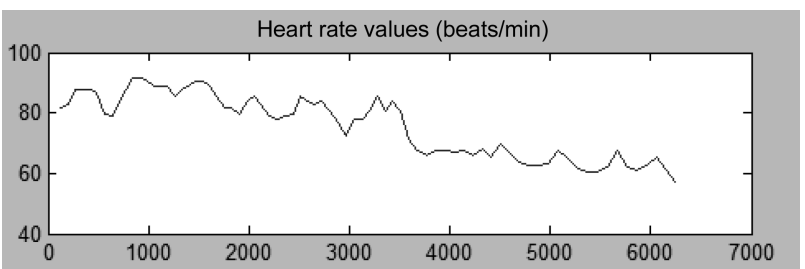

Fig. 7. Example of changing heart rate. 

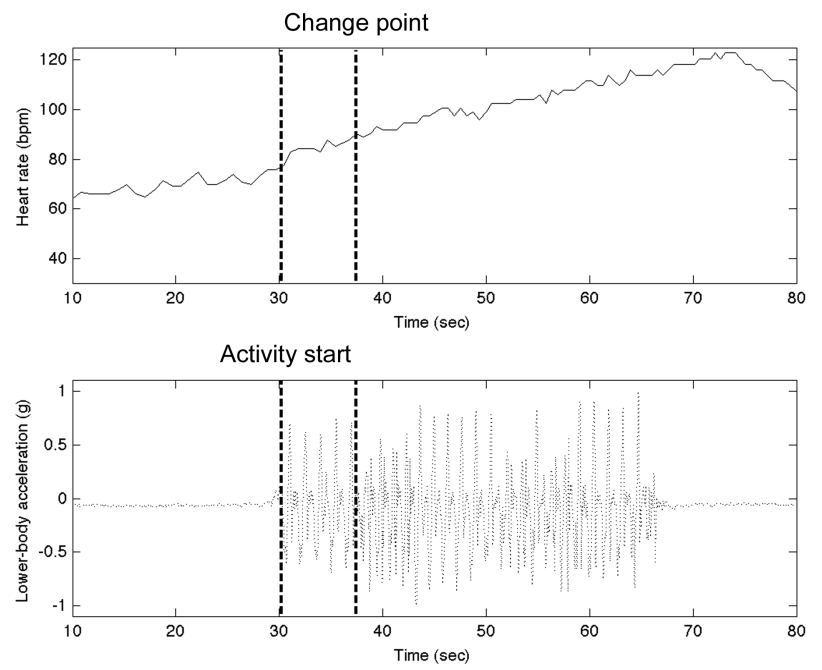

Fig. 8. Example of change of heart rate detected by Cumulative Sum Control Charts (upper graph) correlated with acceleration values (lower graph) for the scenario of changing from no motion to ascending a flight of stairs.

rate, a practical framework that combines both heart rate and activity-related information could be established. If the point at which a change in heart rate occurs can be correlated with a change in activity level, further insight into the behavior of the person being monitored can be provided.

The statistical approach of Cumulative Sum Control Charts (CUSUM) can be used to detect the shift in the mean of a process [21]. In this instance, CUSUM can be applied to the acquired heart rate values. Once a changing pattern has been discovered, this can then be associated with any change in activity levels and hence a further insight into the behavior of the person monitored can be gained.

Fig. 8 presents the synchronised recordings of heart rate and activity data. In this scenario, the inhabitant starts from a 30 second period of being motionless then proceeds to ascend a flight of stairs. The top graph in Fig. 8 presents the recorded heart rate. The initial vertical line on the graph denotes the start of the activity and the second vertical line denotes a change point in the pattern of the heart rate, as detected by the CUSUM algorithm.

The processing of heart rate information in this manner initially aims to detect if there has been a shift in the mean of the heart rate measurements. If this shift is detected, as is the case in the example presented in Fig. 8, this information can be used for cross-referencing purposes with the recorded activity data. Subsequently, the lower graph in Fig. 8, presents the acceleration data acquired from a worn accelerometer during the activity. Examining the acceleration data, it is clearly visible when the activity begins (indicated by the first vertical line). Although there is a degree of latency between the start of the activity and the change point being recognised, it is possible to provide an explanation that the change of heart rate has been caused by a correlated change in activity.

Full details and evaluation of this approach for a range of activities can be found in [22]. Within this evaluation, the CUSUM approach was found to be capable of consistently identifying the changes in heart rate that were correctly corre- (a)
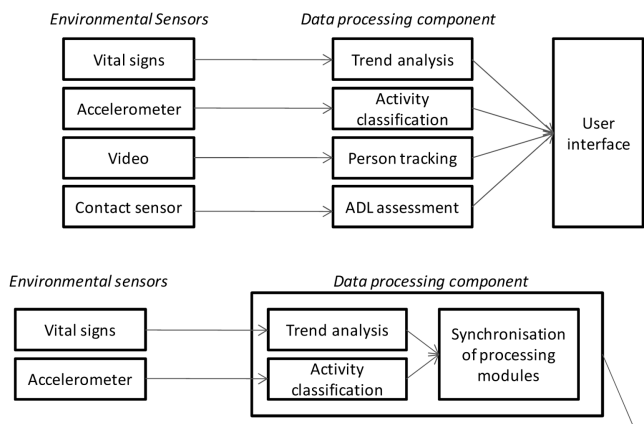

(b)

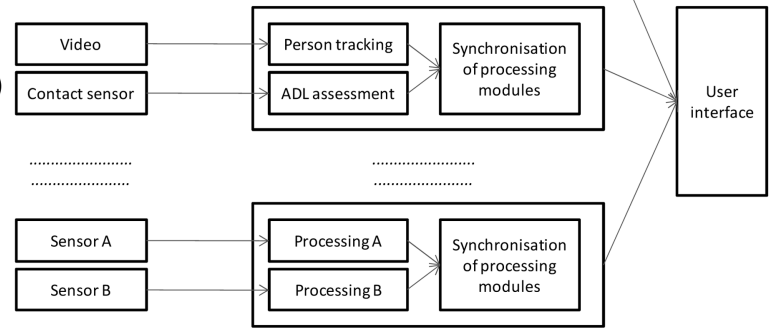

Fig. 9. Management of sensor data (a) conventional mapping of one source of data to one data processing component and (b) proposed augmented architecture with a many to one mapping.

lated with a changes in activity, validated by an accompanying change in the acceleration signal acquired.

\section{CONCLUSION}

In this work we have presented a general overview of the differing types of data that can be recorded in a smart environment. Accompanying this, we have presented a series of challenges that are faced by the data processing component of a smart home, which hinder both the accuracy and reliability of such systems. In an effort to address these challenges, we proposed practical ways in which multiple sources of sensor data can be combined.

Based on the two exemplar case studies, it is apparent that through consideration of additional information, an improvement in the overall accuracy of inferring human behavior and/or wellbeing status can be achieved. We can generalise from these observations and introduce a generic architecture as a means of guidance for how such a practical framework may be established in the future. Fig. 9 presents the differences between the conventional one-to-one mapping from the sensor technology to the data processing component (Fig. 9a) and the many-to-one approach (Fig. 9b) adopted within the two case studies. It is evident that technology has a role to play in offering improvements within home-based care management. The creation of a smart environment within the home not only offers an opportunity to embed sensors in order to acquire information about a person within their own home, it also allows us to process the information collected and subsequently interact with the person while aiming to enhance their underlying quality of life.

To date, research conducted within the AAL domain has provided a deeper insight into factors associated with the design and development of technology to support independent living. This informs future research within this area and moves us one 
step closer to wider deployment of smart home services. Nevertheless, a number of challenges still remain in all of the three main components within the smart home: sensors, data processing and environmental control. From a theoretical perspective, many approaches have provided promising results, however, the performance of the results have deteriorated when tested under non-ideal conditions. As such, further efforts must be made to address the non-ideal situations present when solutions are deployed in situ.

\section{REFERENCES}

1. World Health Organistion, "Ageing," http://www.who.int/topics/ ageing/en/.

2. J. D. Bronzino, The Biomedical Engineering Handbook, 2nd ed., Boca Raton: CRC Press, 1999.

3. Commission of the European Communities, COM (2007) 329 final: proposal for a decision of the European Parliament and of the Council on the participation by the Community in a research and development programme aimed at enhancing the quality of life of older people through the use of new Information and Communication Technologies (ICT), undertaken by several Member States [Brussels, 14.6.2007].

4. AAL Association, "Ambient Assisted Living Joint Programme," http://www.aal-europe.eu.

5. C. D. Nugent, "Delivering care at home through a smarter environment," ATTACH Workshop: Advances in Techniques and Technologies Assisting Care at Home, Dublin, Ireland, 2011.

6. C. D. Nugent, D. D. Finlay, P. Fiorini, Y. Tsumaki, and E. Prassler, "Home automation as a means of independent living," IEEE Transactions on Automation Science and Engineering, vol. 5, no. 1, pp. 1-9, 2008.

7. D. J. Cook and S. K. Das, "How smart are our environments? An updated look at the state of the art," Pervasive and Mobile Computing, vol, 3, no. 2, pp. 53-73, 2007.

8. M. P. Poland, C. D. Nugent, H. Wang, and L. Chen, "Smart home research: projects and issues," International Journal of Ambient Computing and Intelligence, vol. 1, no. 4, pp. 32-45, 2009.

9. T. Kleinberger, M. Becker, E. Ras, A. Holzinger, and P. Muller, "Ambient intelligence in assisted living: enable elderly people to handle future interfaces," Universal Access in Human-Computer Interaction. Ambient Interaction. Lecture Notes in Computer Science Vol. 4555, C. Stephanidis, Ed., Heidelberg: Springer Berlin, pp. 103-112, 2007.

10. B. G. Celler, W. Earnshaw, E. D. Ilsar, L. Betbeder-Matibet, M. F. Harris, R. Clark, T. Hesketh, and N. H. Lovell, "Remote monitoring of health status of the elderly at home. A multidisciplinary project on aging at the University of New South Wales," International Journal of Bio-Medical Computing, vol. 40, no. 2, pp. $147-155,1995$.

11. C. Scanaill, S. Carew, P. Barralon, N. Noury, D. Lyons, and G. Lyons, "A review of approaches to mobility telemonitoring of the elderly in their living environment," Annals of Biomedical Engineering, vol. 34, no. 4, pp. 547-563, 2006.

12. U. Anliker, J. A. Ward, P. Lukowicz, G. Troster, F. Dolveck, M. Baer, F. Keita, E. B. Schenker, F. Catarsi, L. Coluccini, A. Belardinelli, D. Shklarski, M. Alon, E. Hirt, R. Schmid, and M. Vuskovic, "AMON: a wearable multiparameter medical monitoring and alert system," IEEE Transactions on Information Technology in Biomedicine, vol. 8, no. 4, pp. 415-427, 2004.

13. C. Tudor-Locke and L. Lutes, "Why do pedometers work?: a reflection upon the factors related to successfully increasing physical activity," Sports Medicine, vol. 39, no. 12, pp. 981-993, 2009.

14. Z. Shumei, P. McCullagh, C. Nugent, and Z. Huiru, "Activity monitoring using a smart phone's accelerometer with hierarchical classification," The Sixth International Conference on Intelligent Environments, Kuala Lumpur, Malaysia, 2010, pp. 158-163.

15. M. Philipose, K. P. Fishkin, M. Perkowitz, D. J. Patterson, D. Fox, H. Kautz, and D. Hahnel, "Inferring activities from interactions with objects," IEEE Pervasive Computing, vol. 3, no. 4, pp. 50-57, 2004.

16. L. M. Ni, Y. Liu, Y. C. Lau, and A. P. Patil, "LANDMARC: indoor location sensing using active RFID," Wireless Networks, vol. 10, no. 6, pp. 701-710, Nov. 2004.

17. D. Kelly, S. McLoone, and T. Dishongh, "Enabling affordable and efficiently deployed location based smart home systems," Technology and Health Care, vol. 17, no. 3, pp. 221-235, 2009.

18. C. D. Nugent, M. D. Mulvenna, X. Hong, and S. Devlin, "Experiences in the development of a Smart Lab," International Journal of Biomedical Engineering and Technology, vol. 2, no. 4, pp. 319-331, 2009

19. Z. Uhrikova, C. D. Nugent, and V. Hlavac, "The use of computer vision techniques to augment home based sensorised environments," Proceedings of the 30th Annual International Conference of the Engineering in Medicine and Biology Society, Vancouver, Canada, 2008, pp. 2550-2553.

20. P. Bonato, "Wearable sensors and systems," IEEE Engineering in Medicine and Biology Magazine, vol. 29, no. 3, pp. 25-36, MayJun. 2010.

21. A. N. Pettitt, "A simple cumulative sum type statistic for the change-point problem with zero-one observations," Biometrika, vol. 67, no. 1, pp. 79-84, Jan. 1980.

22. L. Galway, S. Zhang, C. Nugent, S. McClean, D. Finlay, and B. Scotney, "Utilizing wearable sensors to investigate the impact of everyday activities on heart rate," Toward Useful Services for Elderly and People with Disabilities. Lecture Notes in Computer Science Vol. 6719, B. Abdulrazak, S. Giroux, B. Bouchard, H. Pigot, and M. Mokhtari, Eds., Heidelberg: Springer Berlin, pp. 184-191, 2011. 


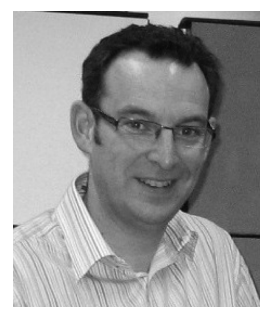

\section{Chris Nugent}

Chris Nugent received a Bachelor of Engineering in Electronic Systems and DPhil in Biomedical Engineering both from the University of Ulster. His current research within biomedical engineering addresses the themes of the development and evaluation of Ambient Assisted Living solutions.

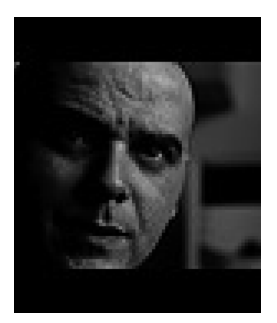

\section{Leo Galway}

Leo Galway received a Bachelor of Science in Computer Science from The Queen's University, Belfast, and both a Master of Science in Computing \& Intelligent Systems and a Ph.D. in Computing \& Information Engineering from the University of Ulster. His current research activity focuses on the design and development of mHealth solutions for assistive living. His research interests include the application of Computational Intelligence techniques within pervasive computing environments and digital entertainment, wearable wireless sensors for assistive living, and mobile software development.

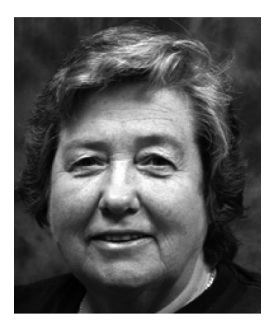

\section{Sally McClean}

Sally McClean is currently Professor of Mathematics in the School of Computing and Information Engineering at the University of Ulster. She took her first degree in Mathematics at Oxford University, then a M.Sc. at Cardiff University in Mathematical Statistics and Operational Research followed by a Ph.D. at the University of Ulster. Her research interests are in mathematical modelling, applied probability, multivariate statistical analysis and applications of mathematical and statistical methods to computer science, particularly database technology. She is a Fellow of the Royal Statistical Society, a member of the IEEE Computer Society, a member of the Operational Research Society, and an Associate Fellow of the Institute of Mathematics and its Applications.

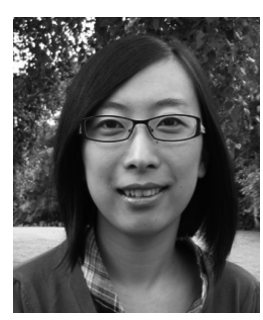

\section{Shuai Zhang}

Shuai Zhang received a Ph.D. degree in intelligent data analysis from the University of Ulster. Her current research interests include knowledge discovery and data integration of semantically heterogeneous aggregate data in a distributed environment, and intelligent data analysis within smart environment and pervasive healthcare technology.

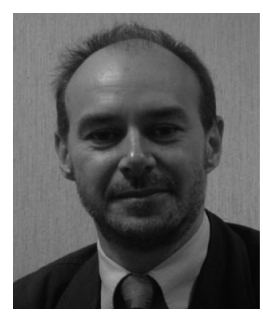

\section{Bryan Scotney}

Bryan Scotney is Professor of Informatics and currently Director of the Computer Science Research Institute at the University of Ulster. He received a BSc in Mathematics from the University of Durham and a PhD in Mathematics from the University of Reading. He has published widely in the area of Knowledge Discovery and Data Mining, focusing on solving the problems of integration of heterogeneous data from distributed sources, particularly where the information available is imprecise and / or uncertain. His early research interest in computational mathematics is now focussed onto the area of digital image processing; this work is concerned mainly with methodological development, but also has application to machine vision and medical imaging. 

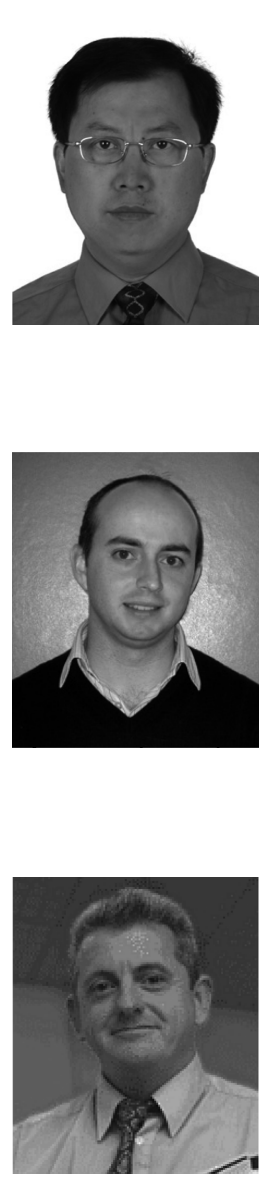

\section{Gerard Parr}

Gerard Parr is Chair in Telecommunications Engineering at the University of Ulster at Coleraine in Northern Ireland. In recent years he has been successful in attracting major EPSRC research funding for a project in Sensing Unmanned Aerial Vehicles that will involve colleagues from UCL, Oxford, UK Home Office, Thales, BAE Systems and Boeing USA. He is PI for the India-UK Advanced Technology Centre (IU-ATC): http://www.iu-atc.com. He was recently invited to become a Member of the Research Councils UK-EPSRC ICT Strategic Advisory Team and was recently appointed to the Technical Advisory Boards for Etisalat-BT Innovation Centre (EBTIC) in Abu Dhabi-UAE and the UK National Centre for Doctoral Training in Communications at the University of Bristol. 